“ (C) 2017 IEEE. Personal use of this material is permitted. Permission from IEEE must be obtained for all other uses, in any current or future media, including

reprinting/republishing this material for advertising or promotional purposes, creating new collective works, for resale or redistribution to servers or lists, or reuse of any copyrighted component of this work in other works." 


\title{
Adaptive Placement for Mobile Sensors in Spatial Prediction under Locational Errors
}

\author{
Linh V. Nguyen, Member, IEEE, Sarath Kodagoda, Member, IEEE, Ravindra Ranasinghe, Member, IEEE and \\ Gamini Dissanayake, Member, IEEE
}

\begin{abstract}
This paper addresses the problem of driving robotic sensors for an energy-constrained mobile wireless network in efficiently monitoring and predicting spatial phenomena, under data locational errors. The paper first discusses how errors of mobile sensor locations affect estimating and predicting the spatial physical processes, given that spatial field to be monitored is modeled by a Gaussian process. It then proposes an optimality criterion for designing optimal sampling paths for the mobile robotic sensors given the localization uncertainties. Although the optimization problem is optimally intractable, it can be resolved by a polynomial approximation algorithm, which is proved to be practically feasible in an energy-constrained mobile sensor network. More importantly, near-optimal solutions of this navigation problem are guaranteed by a lower bound within $1-(1 / e)$ of the optimum. The performance of the proposed approach is evaluated on simulated and real-world data sets, where impact of sensor location errors on the results is demonstrated by comparing the results with those obtained by using noise-less data locations.
\end{abstract}

Index Terms-Mobile robotic wireless sensor networks, spatial prediction, Gaussian processes, locational errors.

\section{INTRODUCTION}

Recently, technological developments in micro-electromechanical systems and wireless communications allow mobile robotic wireless sensor networks (MRWSNs) to have significant impact on monitoring spatial phenomena such as exploring ecosystem change in ocean and on land, observing toxic pollutants and detecting forest fires [1]-[3]. Advantages of the MRWSNs as compared with stationary wireless sensor networks consist of constantly adapting to the changes in the environment and robustly responding to node failures. Moreover, a wireless sensor network incorporating in mobile robotic platforms is capable of being designed strategies to optimally capture the spatial physical phenomena of interest.

In addition to collecting the data, the MRWSNs are also competent to estimate and predict the spatial phenomenon at unobserved locations by combining the available measurements with a model. For instance, in [1] Leonard et al. employed a linear model to predict an ocean field. By defining a graph whose vertices and edges are considered as a single robot's visiting locations and moving paths, respectively, a

This work was partly funded by "predictive analytics for sewer corrosion", which is a collaborative project amongst Sydney Water, Melbourne Water, Water Corporation, South Australian Water, Data 61, University of Newcastle and University of Technology Sydney.

Linh V. Nguyen, Sarath Kodagoda, Ravindra Ranasinghe and Gamini Dissanayake are with Centre for Autonomous Systems, University of Technology Sydney, New South Wales 2007, Australia (e-mail: \{vanlinh.nguyen, sarath.kodagoda, ravindra.ranasinghe, gamini.dissanayake\}@uts.edu.au). path planning algorithm for a mobile robot was proposed in [4] so as to maximize information gain from measurements of a spatio-temporal phenomenon. Choi et al. [5] introduced a Kalman filter based technique to learn the parameters of a physical spatio-temporal process model and then presented criteria to navigate mobile sensors throughout an environment in order to maximize a specified performance. In [6] $\mathrm{Wu}$ et al. proposed a switching scheme for a team of mobile sensors to switch between individual exploration and cooperative exploration as they were exploring an unknown environment. Nevertheless, in most mentioned works, the use of the linear models is disadvantageous as its parameters must be known a priori [7]. Therefore, in this work, we propose to utilize a non-parametric data-driven model, Gaussian process (GP) [8], to statistically model the spatial phenomena.

In the context of a non-parametric model, Suh et al. by their work [9] represented an environmental monitoring navigation strategy for a sensing robot, in which the information gain along the robot's trajectory is maximized. In [10] Cortés proposed a distributed Kriged Kalman filter for robotic wireless sensors in which a gradient based controller was designed to drive the mobile wireless sensors to take optimal samples so that the variance of the estimate error is decreased. Xu et al. primarily used the GP regression for estimating and predicting the generally scalar field and designed optimality criteria based on the Fisher information matrix [11] and the average of the prediction error variances [12] for the optimal sampling paths of the MRWSNs. The authors in [13] introduced the Bayesian optimization based technique for the purpose of choosing the much more relevant informative locations for mobile robotic wireless sensors (MRWSs) in the GP modeled field. However, in most previous works on the spatial prediction in the MRWSNs, there exist two fundamental issues: $(i)$ the networks have been supposed to have the true sensing locations, i.e. location uncertainties are ignored; and (ii) the bounds of solutions of sampling algorithms in energy-constrained MRWSNs have not been analysed.

In fact, precise localization of mobile wireless sensors has been assumed in various applications. For example, in the GP regression, data locations are an essential input component of Gaussian predictive inference. Nonetheless, due to some reasons such as imprecise positioning instruments, coordinate rounding or human based reading error, especially in the MRWSNs where mobility generally increases the uncertainty of sensor nodes, identifying the true sensor locations is very challenging [14]. Moreover, most of the localization algorithms in the wireless sensor networks such as the anchor- 
based method [15] and the beacon-assisted technique [16] generally require the global positioning systems (GPSs). While the GPSs cannot work indoor or in the presence of obstacles [17], it is not cost effective [18]. Inexpensive GPSs normally in turn give significant locational errors. Therefore, in practice, there potentially exist the errors at the sensing locations in the energy-constrained MRWSNs. In the spatial prediction, since the data locations are utilized to compute covariance and crosscovariance matrices and spatial trend, the uncertainties at the sampling locations definitely affect on prediction results.

Motivated by the aforementioned problems, we consider statistical incorporation of the locational errors into the Gaussian spatial prediction and estimation. In fact, this idea has been presented in [2] where Mysorewala et al. introduced a neural network and an extended Kalman filter (EKF) for monitoring environments. The EKF enables the authors to account for the locational errors on results. Nevertheless, their proposed approach is based on a parametric radial basis function model, which is not really flexible with respect to highly complex real-world physical fields.

As a result, in this paper, we present how the errors at the data locations are statistically incorporated into predicting and estimating the spatial fields modeled by a non-parametric GP. Further, the fundamental scheme monitoring and predicting spatial fields using the MRWSNs is to informatively find the optimal navigation. Thus, we formulate an adaptive sampling optimization problem in terms of minimum average variance criteria, where sensor location uncertainties are comprised. This combinatorial NP-hard sampling optimality criterion is then improved to significantly reduce computational time in greedy algorithm implementation. Moreover, the near-optimal solutions of the sampling optimization obtained by a greedy algorithm are proved to be guaranteed by a lower bound that performs a level $1-(1 / e)$ of the optimal solution. An upper bound for any other methods can be found from our nearoptimal solution.

The remainder of this paper is organized as follows. Section II discusses a predictive inference approach for the spatial field where the errors at sensor locations are considered. To enhance quality of spatial prediction, a sampling strategy for the MRWSs is designed in Section III. A bound of solutions is also presented in this section. Sections IV evaluates the proposed approach by simulation results before conclusions are drawn in Section V.

\section{Spatial PREDictive InfEREnCE Under LocAtional ERRORS}

In most applications of environmental monitoring using the WSNs [19]-[21] we suppose that devices that are utilized to measure the sensor locations work impeccably. Nonetheless, in practice, there exist availabilities of spatial uncertainty associated with those data locations. On the other hand, as represented in our previous work [21], the data locations are significantly employed to compute the covariates at locations, as well as the spatial covariance function. In equivalent words, the spatial prediction cannot be obtained without the sensing positions. This shows that the location errors have potentially serious effect on results of model parameter estimation and prediction quality. Hence, in this section, we introduce how the errors of the measuring locations affect on estimating and predicting the spatial phenomena.

Consider a mobile network of $N$ identical sensors, indexed by $i \in\{1, \ldots, N\}$, spatially distributed in the spatial field of interest $\mathcal{Q} \subset \mathbb{R}^{d}$, where each mobile sensor can gather data at times $t \in \mathbb{Z}_{>0}$. Let $s_{t, i}$ denote an intended location for a mobile sensor $i$ at time step $t$, identified by a positioning instrument. Due to imprecision of the device, we define $r_{t, i}$ as a real but unidentified location where the sensor is actually positioned. As discussed in [22], a mobile agent's location error is given by

$$
r_{t, i}=s_{t, i}+\gamma_{t, i}
$$

where $\gamma_{t, i}$ is a locational error. In two dimension environments, $\gamma_{t, i}$ is supposed to be an uncorrelated bivariate normal distribution with a zero mean and a variance $\sigma_{e}^{2}$ as specified by

$$
\gamma_{t, i} \sim \mathcal{N}\left(\mathbf{0}, \sigma_{e}^{2} I_{2}\right),
$$

where $I_{2}$ is a $2 \times 2$ identity matrix. Note that errors at different locations are independent, and $\gamma_{t, i}$ is independent of $r_{t, i}$ and $s_{t, i}$. We assume that $\gamma_{i}=\gamma_{t, i}$ is constant over time. If $f\left(\gamma_{i}\right)$ denotes a probability density function of $\gamma_{i}=\left(\gamma_{i, x}, \gamma_{i, y}\right)$, it is specified as

$$
f\left(\gamma_{i}\right)=\frac{1}{2 \sigma_{e}^{2}} \exp \left(-\frac{\gamma_{i, x}^{2}+\gamma_{i, y}^{2}}{2 \sigma_{e}^{2}}\right) .
$$

By the use of the spatial field model introduced in [21], the spatial process gathered by the sensor $i$ at time instant $t$ is now formally described under consideration of the location errors as follows

$$
y_{t, i}^{*}=X\left(s_{t, i}+\gamma_{i}\right) \beta_{t}^{*}+\xi\left(s_{t, i}+\gamma_{i}\right)+\varepsilon\left(s_{t, i}+\gamma_{i}\right),
$$

where $y_{t, i}^{*}$ is a measurement taken at $r_{t, i}$ but erroneously positioned at $s_{t, i} \cdot \xi\left(s_{t, i}+\gamma_{i}\right)$ is a zero mean stationary GP, $\varepsilon\left(s_{t, i}+\gamma_{i}\right)$ is an independent and identically distributed noise with a zero mean and a variance $\left(\tau_{t}^{*}\right)^{2}$. $X\left(s_{t, i}+\gamma_{i}\right)$ is covariates at a location $r_{t, i}$ and $\beta_{t}^{*}$ is a vector of $p$ mean parameters. Both $\beta_{t}^{*}$ and $\left(\tau_{t}^{*}\right)^{2}$ are learned by incorporating the errors in locations into data locations. Since means of $\xi\left(s_{t, i}+\gamma_{i}\right)$ and $\varepsilon\left(s_{t, i}+\gamma_{i}\right)$ are zero, expectation of $y_{t, i}^{*}$, $\mu^{*}\left(s_{t, i}\right)$, can be given as

$$
\mu^{*}\left(s_{t, i}\right)=\mathbb{E}\left(y_{t, i}^{*}\right)=\left(\int X\left(s_{t, i}+\gamma_{i}\right) f\left(\gamma_{i}\right) d \gamma_{i}\right) \beta_{t}^{*} .
$$

Now, we consider how to compute the covariance function under presence of the locational uncertainties. Because the data locations participate in the covariance function, under distribution of the locational errors the covariance value between $s_{t, i}$ and $s_{t, j}$, affected by the uncertainties of locations, can be easily derived by

$$
\begin{aligned}
& \operatorname{cov}^{*}\left(s_{t, i}, s_{t, j}\right)=\operatorname{cov}\left(s_{t, i}+\gamma_{i}, s_{t, j}+\gamma_{j}\right) \\
& \quad=\iint \operatorname{cov}\left(s_{t, i}-s_{t, j}+\gamma_{i}-\gamma_{j}\right) f\left(\gamma_{i}\right) f\left(\gamma_{j}\right) d \gamma_{i} d \gamma_{j},
\end{aligned}
$$

where $\operatorname{cov}(\cdot)$ is a covariance function [23]. 
The variance of the spatial process at a location $s_{t, i}$ as shown by Cressie et al. in [24] includes three components of spatial dependence, measurement error and trend, specified by

$$
\operatorname{var}^{*}\left(s_{t, i}\right)=\operatorname{cov}(\mathbf{0})+\left(\tau_{t}^{*}\right)^{2}+\left(\beta_{t}^{*}\right)^{T} T\left(s_{t, i}\right) \beta_{t}^{*} .
$$

Element of the spatial dependence is clearly computed by the covariance function, $\operatorname{cov}(\mathbf{0})$, where $\mathbf{0}$ is a zero vector. The measurement error $\left(\tau_{t}^{*}\right)^{2}$ can be estimated by measurements. And, since the locational error impacts on covariates of computing the spatial trend [21], this error is also transferred to the variance. Moreover, element of the spatial trend is described by $\left(\beta_{t}^{*}\right)^{T} T\left(s_{t, i}\right) \beta_{t}^{*}$, where $T\left(s_{t, i}\right)$ is given by

$$
\begin{gathered}
T\left(s_{t, i}\right)=\int X\left(s_{t, i}+\gamma_{i}\right) X\left(s_{t, i}+\gamma_{i}\right)^{T} f\left(\gamma_{i}\right) d \gamma_{i} \\
-\left(\int X\left(s_{t, i}+\gamma_{i}\right) f\left(\gamma_{i}\right) d \gamma_{i}\right)\left(\int X\left(s_{t, i}+\gamma_{i}\right) f\left(\gamma_{i}\right) d \gamma_{i}\right)^{T} .
\end{gathered}
$$

Notice that if $\gamma_{i} \equiv \mathbf{0}$, then $T\left(s_{t, i}\right)=0$.

It can be obviously seen that integrating equations (3), (4) and (6) is analytically intractable. As a consequence, these equations can be numerically estimated by the use of Monte Carlo approaches [25]. The idea behind the Monte Carlo methods is to draw a number of samples for the distribution of $f\left(\gamma_{i}\right)$, then the integral can be approximated by averaging samples of the function inside the integral at corresponding sampled points of $f\left(\gamma_{i}\right)$.

In the following, we present how to estimate and predict the spatial process under incorporation of sensing location errors. Let $z^{*}(w)$ (hereafter $z^{*}$ ) denote the spatial variables at unobserved locations $w=\left(w_{1}^{T}, w_{2}^{T}, \ldots, w_{m}^{T}\right)^{T}$. We define collection of all identified sensor locations and collective measurements from time 1 to $t$ as $s_{1: t}$ (correspondingly real and unknown locations $r_{1: t}$ ) and $y_{1: t}^{*}$. Given $y_{1: t}^{*}$ measured at $r_{1: t}$ but erroneously at $s_{1: t}$, we expect to efficiently predict $z^{*}(w)$. Using the principle of the scheme in [21], the posterior distribution of the random variables $z^{*}$ is Gaussian, described by

$$
z^{*} \mid y_{1: t}^{*} \sim \mathcal{N}\left(\mu_{z^{*} \mid y_{1: t}^{*}}, \Sigma_{z^{*} \mid y_{1: t}^{*}}\right)
$$

where the vector of the conditional mean is given as

$$
\mu_{z^{*} \mid y_{1: t}^{*}}=X(w) \beta_{t}^{*}+\Sigma_{z^{*} y_{1: k}^{*}}\left(\Sigma_{1: t}^{*}\right)^{-1}\left(y_{1: t}^{*}-X^{*}\left(s_{1: t}\right) \beta_{t}^{*}\right),
$$

and the matrix of the conditional covariances is specified as

$$
\Sigma_{z^{*} \mid y_{1: t}^{*}}=\Sigma_{z^{*} z^{*}}-\Sigma_{z^{*} y_{1: t}^{*}}\left(\Sigma_{1: t}^{*}\right)^{-1} \Sigma_{y_{1: t}^{*} z^{*}} .
$$

In equations (8) and (9), $X(w)$ is a $m \times p$ matrix of covariates at the unmeasured locations $w$, and the matrix $\Sigma_{z^{*} y_{1 . t}^{*}}$ $\left(=\left(\Sigma_{y_{1: t}^{*} z^{*}}\right)^{T}\right)$ is a $m \times t N$ cross-covariance matrix between $z^{*}$ and $y_{1: t}^{*} . \Sigma_{z^{*} z^{*}}$ is a $m \times m$ covariance matrix of $z^{*}$. $\Sigma_{1: t}^{*}$ is a $t N \times t N$ covariance matrix of $y_{1: t}^{*}$ of which off-diagonal elements can be computed by (4) and diagonal elements can be obtained by (5). Each row of $X^{*}\left(s_{1: t}\right)$ can be obtained by $\int X\left(s_{t, i}+\gamma_{i}\right) f\left(\gamma_{i}\right) d \gamma_{i}$.

In order to illustrate the effect of the presence of sensor location errors on the spatial prediction, we utilize a true spatial field in Fig. 1, where the field is generated by a

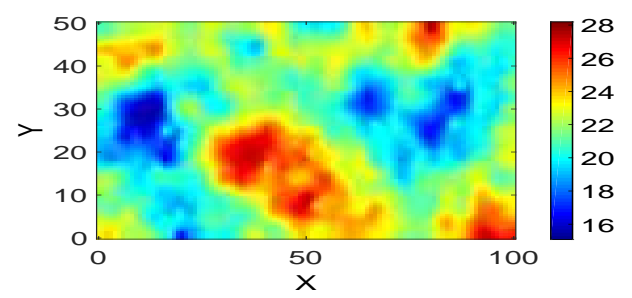

Fig. 1: The true field of numerically generated data.

GP model with a mean of $\beta=20$. A marginal variance $\sigma^{2}$, a range parameter $\phi$, a noise variance $\tau^{2}$ and a Matérn smoothness of a Matérn covariance function are set to 4.0, 7.0, 0.1 and 1, respectively. After gathering 50 measurements, we first estimate the parameters of the spatial model by the use of the maximum likelihood technique [26], and results are demonstrated in Table I. The third column represents the estimated parameters without locational errors and the columns right to it represent the estimated parameters with different errors. Here, three uncorrelated bivariate normal distributions of the location errors with a similar zero mean and variances $\sigma_{e}^{2}$ of $1^{2}, 2^{2}$ and $3^{2}$ are studied. It can be seen that the estimations of mean parameters and marginal variances are minimally affected by the locational errors. On the contrary, estimated range parameters are significantly affected by these errors. This leads to decrease of correlations of random variables in the field, which potentially affects the predictions at unobserved locations. Empirically using the model parameters, we then predict the physical phenomena at unmeasured locations on a $100 \times 100$ grid covering the whole region. Four scenarios are considered. Firstly, the posterior means are predicted, assuming no locational errors. The predicted field is demonstrated in Fig. 2a. Secondly, the predicted means with different location errors are analyzed. The corresponding predicted means are shown in Figures $2 b$, $2 \mathrm{c}$ and $2 \mathrm{~d}$, respectively. Generally, the predicted fields in Fig. 2 are comparable with the true field in Fig. 1. However, the contrast between them is obvious. For instance, when zero or small locational errors are incorporated into the predictions, there are much variation on surface of the field in Figures 2a and $2 \mathrm{~b}$, which reasonably match with the true field. In contrast, as the increase of the uncertainties in locations, the surface of the predicted field becomes smoother and abstract.

\section{AdAPtive SAmpling Under Locational ERRORS}

One of the fundamental questions in the MRWSNs is to drive the mobile sensors to effectively sample the phenomena. In other words, given a limited number of the mobile agents, there is a need to design optimal sampling paths so that sensing robots can capture the physical environment at the most informative locations. And, eventually, the network converges to the best estimation of the model parameters and the best prediction of the field at unobserved locations. More importantly, under the presence of the errors in sensing locations, these requirements are more complicated. Therefore, in this section, we present a strategy for the MRWSs at time step $t$ to be able to adaptively sample the field at time instant 
TABLE I: PARAMETER ESTIMATION

\begin{tabular}{|c|c|c|c|c|c|}
\hline \multirow{3}{*}{ Parameters } & \multirow{3}{*}{ Real values of parameters } & \multicolumn{4}{|c|}{ Estimated model parameters } \\
\hline & & \multirow{2}{*}{$\begin{array}{l}\text { Without } \\
\text { location } \\
\text { errors }\end{array}$} & \multicolumn{3}{|c|}{$\begin{array}{l}\text { With location errors of zero mean and } \\
\text { standard deviation }\end{array}$} \\
\hline & & & $\sigma_{e}=1$ & $\sigma_{e}=2$ & $\sigma_{e}=3$ \\
\hline $\bar{\beta}$ & 20.000 & 21.231 & 21.244 & 21.374 & 21.348 \\
\hline$\sigma^{2}$ & 4.000 & 4.558 & 4.435 & 4.260 & 4.519 \\
\hline$\phi$ & 7.000 & 8.296 & 8.437 & 9.734 & 10.893 \\
\hline$\tau^{2}$ & 0.100 & 0.093 & 0.007 & 0.00001 & 0.00001 \\
\hline
\end{tabular}

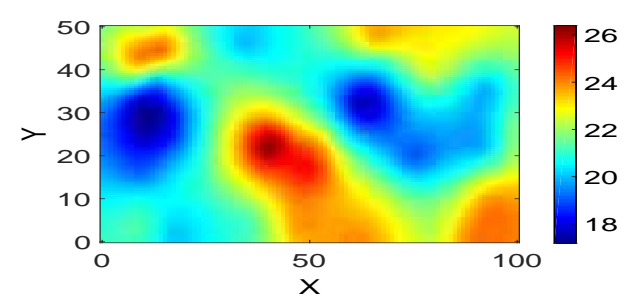

(a)

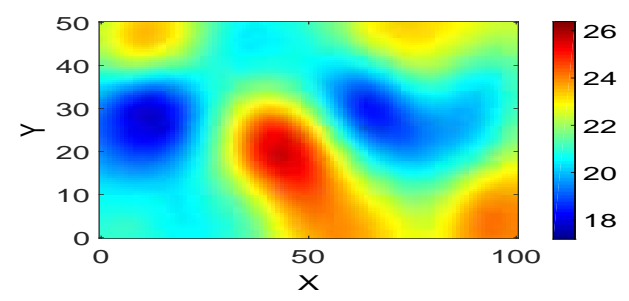

(c)

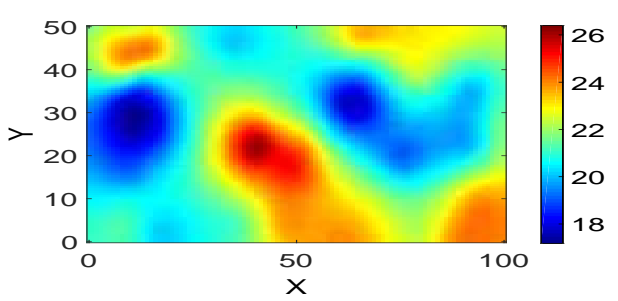

(b)

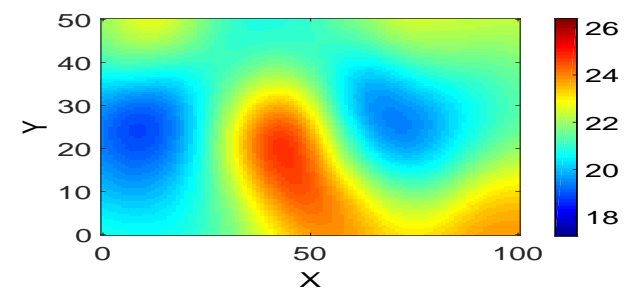

(d)

Fig. 2: Predicted field using 50 measurements (a) without locational errors, (b) $\sigma_{e}^{2}=1^{2}$, (c) $\sigma_{e}^{2}=2^{2}$ and (d) $\sigma_{e}^{2}=3^{2}$.

$t+1$. The strategy is studied under the effect of the sensor location errors.

\section{A. One-step-ahead Inference}

It can be clearly seen that looking for the most informative locations $s_{t+1}$ at time instant $t+1$ without $y_{t+1}$ or $y_{t+1}^{*}$ is intractable. Hence, in order to address this problem, the one-step-ahead forecast is utilized to predict the latent spatial values at time $t+1$, given the collective observations up to time $t$. The measurements collected by $N$ MRWSs at time step $t+1$ can be represented as

$$
y_{t+1}^{*}=z_{t+1}^{*}+\varepsilon,
$$

where $\varepsilon \sim \mathcal{N}\left(\mathbf{0},\left(\tau_{t}^{*}\right)^{2} I_{t+1}\right)$ is a vector of independent and identically distributed noises, and $I_{t+1}$ is a $N \times N$ identity matrix. $z_{t+1}^{*}$ are noise-free observations (also known as latent random field). Given observations under the presence of the locational errors up to time $t, y_{1: t}^{*}$, the latent random process at time step $t+1$ can be forecasted by

$$
\widehat{z_{t+1}^{*}}\left|y_{1: t}^{*}=z_{t}^{*}\right| y_{1: t}^{*}+\eta_{t+1} \text {, }
$$

where $z_{t}^{*} \mid y_{1: t}^{*}$ is the random field at time $t$ and can be computed by (7). $\eta_{t+1} \sim \mathcal{N}\left(\mathbf{0}, \sigma_{\text {sys }}^{2} I_{t+1}\right)$ and $\sigma_{\text {sys }}^{2}$ is a system error that describes the changes in the elements of the parameters vector between times $t$ and $t+1$. Therefore, the forecasted measurements at time $t+1$ conditioned on $y_{1: t}^{*}$ are

$$
\widehat{y_{t+1}^{*}} \mid y_{1: t}^{*} \sim \mathcal{N}\left(\mu_{t+1}^{*}, \Sigma_{t+1}^{*}\right),
$$

where the posterior mean vector, $\mu_{t+1}^{*}$, can be obtained by applying (8), and the posterior covariance matrix is

$$
\begin{aligned}
\Sigma_{t+1}^{*}= & \Sigma_{y_{t+1}^{*} y_{t+1}^{*}}-\Sigma_{y_{t+1}^{*} y_{1: t}^{*}}\left(\Sigma_{1: t}^{*}\right)^{-1} \Sigma_{y_{1: t}^{*} y_{t+1}^{*}} \\
& +\left(\sigma_{\text {sys }}^{2}+\left(\tau_{t}^{*}\right)^{2}\right) I_{t+1} .
\end{aligned}
$$

In (13), $\Sigma_{y_{t+1}^{*} y_{t+1}^{*}}$ is a $N \times N$ covariance matrix of $y_{t+1}^{*}$, $\Sigma_{y_{t+1}^{*} y_{1: t}^{*}}\left(=\left(\Sigma_{y_{1: t}^{*} y_{t+1}^{*}}\right)^{T}\right)$ is a $N \times t N$ cross-covariance matrix between $y_{t+1}^{*}$ and $y_{1: t}^{*}$. Both $\Sigma_{y_{t+1}^{*} y_{t+1}^{*}}$ and $\Sigma_{y_{t+1}^{*} y_{1: t}^{*}}$ are calculated based on next locations $s_{t+1}$ with the locational error $\gamma_{i}$ for each sensor.

\section{B. Navigation Strategy}

In order to design the sampling navigation for the mobile sensor network, we first discretize the spatial field $\mathcal{Q}$ into $V$ spatial areas of interest with small neighbours. We define $M$ as cardinality of $V$. Let $S$ and $Z$ denote the set of locations and the vector of latent variables at circumcentres [27] of $V$ spatial areas. We then address the optimization of the sampling paths for the MRWSNs that approximately drive the mobile agents on the lattice of vertices at the circumcentres of $V$. The optimal sampling scheme aims to minimize the uncertainties at all unmeasured locations over the region. It can be noted that the discretization of the field guarantees that $M \gg N$.

Now, let us consider the uncertainties at unobserved locations of $S$. If $t N \ll M$, that is the number of locations that the mobile sensors have visited is trivial as compared with $M$, the covariance matrix of the random variables $Z$, conditioned on 
collective measurements up to time $t+1, y_{1: t+1}^{*}=y_{1: t}^{*} \cup \widehat{y_{t+1}^{*}}$, can be predicted by

$$
\Sigma_{Z \mid y_{1: t+1}^{*}}=\Sigma_{Z Z}-\Sigma_{Z y_{1: t+1}^{*}}\left(\Sigma_{1: t+1}^{*}\right)^{-1} \Sigma_{y_{1: t+1}^{*} Z},
$$

where

$$
\Sigma_{1: t+1}^{*}=\left[\begin{array}{cc}
\Sigma_{1: t}^{*} & \Sigma_{y_{1: t}^{*} y_{t+1}^{*}} \\
\Sigma_{y_{t+1}^{*} y_{1: t}^{*}} & \Sigma_{t+1}^{*}
\end{array}\right],
$$

and $\Sigma_{Z y_{1: t+1}^{*}}\left(=\left(\Sigma_{y_{1: t+1}^{*} Z}\right)^{T}\right)$ is a $M \times t(N+1)$ crosscovariance matrix between $Z$ and $y_{1: t+1}^{*}$. In equivalent words, $\Sigma_{Z y_{1: t+1}^{*}}$ can also be evaluated by

$$
\Sigma_{Z y_{1: t+1}^{*}}=\left[\begin{array}{ll}
\Sigma_{Z y_{1: t}^{*}} & \Sigma_{Z y_{t+1}^{*}}
\end{array}\right] .
$$

Though we do not have the model parameters at time $t+1$, both $\Sigma_{Z y_{1: t}^{*}}$ and $\Sigma_{Z y_{t+1}^{*}}$ can be obtained based on the hyperparameters learned at $t$. In order to maximize the quality of prediction at all unmeasured locations, the sampling problem is formulated into finding the next sensor locations $s_{t+1}$ at time $t+1$ so that average of trace of the matrix $\Sigma_{Z \mid y_{1: t+1}^{*}}$ is minimized. In other words, the formulated problem in the sampling strategy is to minimize the average of variances at all unobserved locations. Since $\Sigma_{Z Z}$ is dependent on the locations in $S$ and the model parameters learned at current step, $\Sigma_{Z Z}$ is constant at time step $t$. Consequently, the problem of the sampling strategy can be represented as

$$
s_{t+1}^{o p t}=\underset{s_{t+1, i} \in S_{i}^{(t)}}{\operatorname{argmax}} \frac{1}{M} \operatorname{tr}\left(\Sigma_{Z y_{1: t+1}^{*}}\left(\Sigma_{1: t+1}^{*}\right)^{-1} \Sigma_{y_{1: t+1}^{*} Z}\right),
$$

where $S_{i}^{(t)} \subset S$ is a discrete set of locations bounded by $\Omega_{i}^{(t)}$. Here, $\Omega_{i}^{(t)}$ is the allowable movement region of robotic sensor $i$ at time $t+1$ [21]. $\Omega_{i}^{(t)}$ is developed based on the contraction and Voronoi concepts [27], which preserve the safe navigation of the MRWSNs, and the maximum distance a mobile sensor can move between time instances.

Selecting an optimal subset $s_{t+1}^{o p t}$ out of all potential locations in $S$ to optimize the problem (15), as proved by Ko et al. [28], is combinatorial NP-hard. This optimization can often be effectively resolved by a near-optimal greedy heuristic algorithm. Nonetheless, since $M \gg N$, the energyconstrained networks of mobile robotic wireless sensors can intractably run the greedy algorithm to address the problem (15). This computational complexity can be demonstrated by the following theorem.

Theorem 1: The greedy algorithm can resolve the optimization problem (15) in time $\mathcal{O}\left(t M^{2} N^{2}\right)$.

Proof: It can be seen that the main cost of the problem (15) is to compute multiplying $\Sigma_{Z y_{1: t+1}^{*}}\left(\Sigma_{1: t+1}^{*}\right)^{-1} \Sigma_{y_{1: t+1}^{*} Z}$. This cost requires $\mathcal{O}\left(t M^{2} N\right)$ operations. Furthermore, in the adaptive sampling navigation scheme, there is a need to find $N$ next sensor locations at every time instant. This makes the greedy algorithm run $N$ iterations in time $\mathcal{O}(N)$. Hence, the optimization problem (15) can be resolved in computing time $\mathcal{O}\left(t M^{2} N^{2}\right)$.

Due to the intractability of the problem (15) in the energyconstrained MRWSNs, in this study, we enhance the feasibility of this optimization by using one of properties of trace of a product of matrices. Note that as shown in [29], $\operatorname{tr}(A B)=\operatorname{tr}(B A)$. As a result, the optimality criterion (15) can be restated as follows.

$$
s_{t+1}^{o p t}=\underset{s_{t+1, i} \in S_{i}^{(t)}}{\operatorname{argmax}} \frac{1}{M} \operatorname{tr}\left(\Sigma_{y_{1: t+1}^{*} Z} \Sigma_{Z y_{1: t+1}^{*}}\left(\Sigma_{1: t+1}^{*}\right)^{-1}\right),
$$

The equivalent optimization problem (16) is more tractable.

Theorem 2: The near-optimal solution of the optimality criterion (16) can be obtained by a greedy heuristic algorithm in time $\mathcal{O}\left(t M N^{3}\right)$.

Proof: The proof is similar and referred to the proof of the Theorem 1. However, it is noted that the computational complexity of matrix $\Sigma_{y_{1: t+1}^{*}} z \Sigma_{Z y_{1: t+1}^{*}}\left(\Sigma_{1: t+1}^{*}\right)^{-1}$ is $\mathcal{O}\left(t M N^{2}\right)$.

This new proposition in turn gives a significant benefit in terms of computation to the mobile sensor networks. The effectiveness of this improvement will be illustrated in the result sections.

It is to be noted that the location $s_{t+1, i}^{o p t}$ for the $i^{t h}$ mobile sensor at time $t+1$ must be selected from one of all potential $s_{t+1, i}$ points inside $S_{i}^{(t)}$ bounded by $\Omega_{i}^{(t)}$. The solution of (16) is obtained if all $s_{t+1, i}^{o p t}$ locations are found in the corresponding regions $\Omega_{i}^{(t)}$. Nevertheless, $s_{t+1}^{o p t}$ cannot be really found due to the NP-hard complete of the problem (16). Here, we present an efficient algorithm which approximately finds the near-optimal sampling points $\bar{s}_{t+1}$ for the mobile sensors by greedily adding the next sensor locations.

1) Algorithmic approach: Let $Z_{\Omega_{i}^{(t)}}$ denote the vector of the variables at unobserved spatial sites inside $\Omega_{i}^{(t)}$ and $Z_{\Omega^{(t)}}=\bigcup_{i=1}^{N} Z_{\Omega_{i}^{(t)}}$ denote the variables at unmeasured locations in all the allowable movement regions of a $N$ mobile sensors network. We also define $S^{(t)}=\bigcup_{i=1}^{N} S_{i}^{(t)}$.

At each time step $t$, it starts from an empty set of locations, $\bar{s}_{t+1}=\oslash$, that corresponds to the empty set of predicted measurements at time step $t+1, \bar{y}_{t+1}^{*}=\oslash$. We first compute

$$
\frac{1}{M} \operatorname{tr}\left(\Sigma_{y_{1: t+1}^{*}} Z \Sigma_{Z y_{1: t+1}^{*}}\left(\Sigma_{1: t+1}^{*}\right)^{-1}\right),
$$

where $\widehat{y_{t+1}^{*}}=\left\{\widehat{y}_{t+1, j}^{*}\right\}$, and $\widehat{y}_{t+1, j}^{*} \in Z_{\Omega^{(t)}}$. Note that $\widehat{y}_{t+1, j}^{*}$ can be found by applying (12). It returns a vector of average traces. Choose the maximum value from this vector, we then easily obtain $\bar{y}_{t+1, j}^{*}$ and the corresponding location $\bar{s}_{t+1, j}$. If $\bar{s}_{t+1, j}$ is inside $\Omega_{i}^{(t)}$, it is certainly the next location for sensor $i$. Both $\bar{y}_{t+1, j}^{*}$ and $\bar{s}_{t+1, j}$ are then added to $\bar{y}_{t+1}^{*}$ and $\bar{s}_{t+1}$, respectively; and the latent variable vector $Z_{\Omega_{i}^{(t)}}$ and the set $S_{i}^{(t)}$ in $\Omega_{i}^{(t)}$ are removed from $Z_{\Omega^{(t)}}$ and $S^{(t)}$. Iteratively, we run this iteration of the algorithm until $\bar{s}_{t+1}$ reaches to cardinality, $N$. At each iteration, the solution $\bar{y}_{t+1, j}^{*}$ and the corresponding location $\bar{s}_{t+1, j}$ found are greedily added to $\bar{y}_{t+1}^{*}$ and $\bar{s}_{t+1}$, respectively. If the current resulting location $\bar{s}_{t+1, j}$ is identified as an element of $S_{i}^{(t)}$, then $S_{i}^{(t)}$ and the corresponding vector $Z_{\Omega_{i}^{(t)}}$ are removed from $S^{(t)}$ and $Z_{\Omega^{(t)}}$.

The navigation strategy is algorithmically summarized in Algorithm 1. 


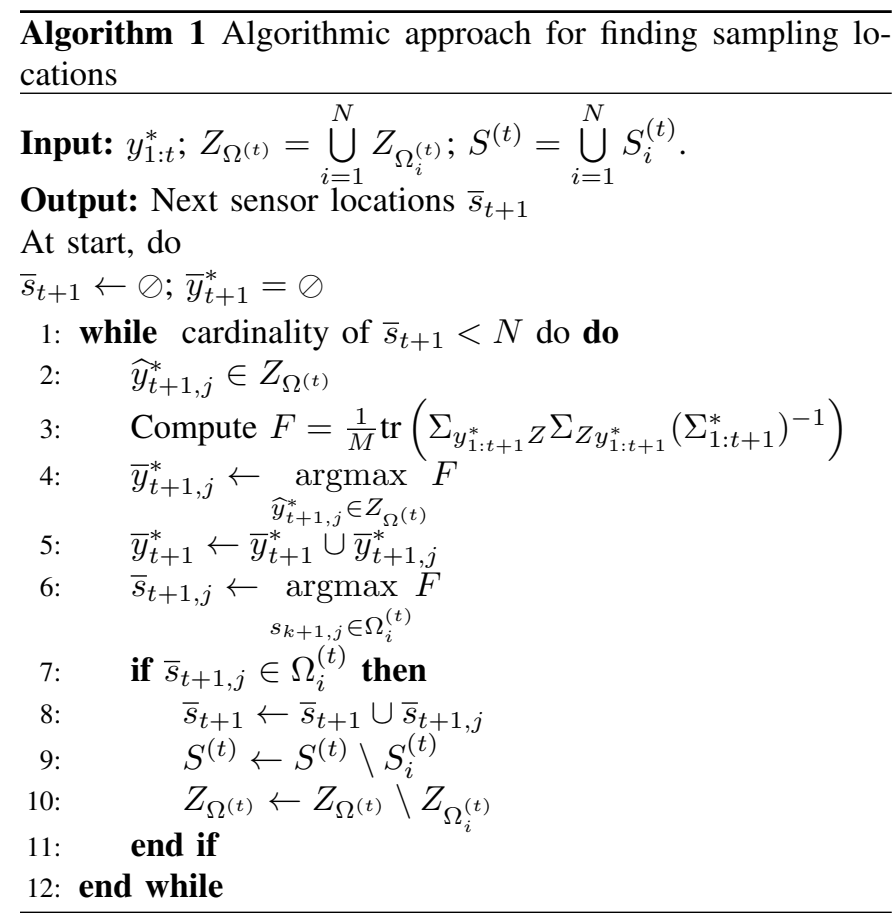

\section{A Solution Bound}

As discussed in the previous subsection III-B, finding the optimal solution $s_{t+1}^{o p t}$ for the proposed criterion (16) is intractable, particularly in the energy-constrained robotic sensor networks. Nonetheless, a near-optimal but not yet guaranteed solution can be obtained by a greedy heuristic algorithm in polynomial time. In this section, we introduce a bound for the found solution.

As presented by Nemhauser et al. in [30], a greedily resolved solution can be bounded if the set function in (16) holds both the monotonicity and submodularity properties. Therefore, we first define

$$
F\left(s_{t+1}\right)=\frac{1}{M} \operatorname{tr}\left(\Sigma_{y_{1: t+1}^{*}} Z^{\Sigma_{Z y_{1: t+1}^{*}}}\left(\Sigma_{1: t+1}^{*}\right)^{-1}\right),
$$

where $s_{t+1}$ is the set of next locations of mobile agents at time $t+1$, and show that the set function $F\left(s_{t+1}\right)$ is monotonic and submodular.

Lemma 3: The set function $s_{t+1} \mapsto F\left(s_{t+1}\right)$ is monotonic.

Proof: We let $A=\Sigma_{y_{1: t+1}^{*}} \Sigma_{Z y_{1: t+1}^{*}}, B=\left(\Sigma_{1: t+1}^{*}\right)^{-1}$ and $W=A B$. If we can show that $W$ is positive semi-definite, $\operatorname{tr}(W)$ is monotonic.

It can be clearly seen that since $\Sigma_{y_{1: t+1}^{*} Z}$ and $\Sigma_{1: t+1}^{*}$ are cross-covariance and covariance matrices, $A$ and $B$ are positive semi-definite. Let $P=\left(A^{\frac{1}{2}}\right)^{-1} A B A^{\frac{1}{2}}$. Because $A$ is non-singular, $A^{\frac{1}{2}}$ is non-singular. As a consequence, $W$ and $P$ are similar. $P$ can be rewritten as $P=A^{\frac{1}{2}} B A^{\frac{1}{2}}$. Since $B$ is positive semi-definite, then $P$ is also positive semi-definite. As illustrated in [29] that if $W$ and $P$ are similar, they have the same eigenvalues. Therefore, having the same eigenvalues of the positive semi-definite matrix $P$ proves that $W$ is positive semi-definite.

Suppose that we increase the set $s_{t+1}$ by one sensor location, $s_{t+1}^{\prime}=s_{t+1} \cup s_{t+1, j}$, then size of the square matrix
$W$ is increased by one. As the eigenvalues of $W$ is nonnegative, $F\left(s_{t+1}^{\prime}\right) \geq F\left(s_{t+1}\right)$, which completes the proof.

Lemma 4: The set function $s_{t+1} \mapsto F\left(s_{t+1}\right)$ is submodular.

Proof: It is known that $\left|s_{t+1}\right|=N$. If $N=0$ (at beginning of the greedy algorithm when $s_{t+1}$ is empty), we define the size of the square matrix $W$ as $k \times k$. Therefore, the size of $W$ with respect to $F\left(s_{t+1}\right)$ is $(k+N) \times(k+N)$, and the size of $W$ with respect to $F\left(s_{t+1}^{\prime}\right)$ is $(k+N+1) \times(k+N+1)$. Notice that $s_{t+1}^{\prime} \cup s_{t+1}=s_{t+1}^{\prime}$ and $s_{t+1}^{\prime} \cap s_{t+1}=s_{t+1, j}$. Similarly, the size of $W$ with respect to $F\left(s_{t+1}^{\prime} \cup s_{t+1}\right)$ is $(k+N+1) \times(k+N+1)$, and the size of $W$ with respect to $F\left(s_{t+1}^{\prime} \cap s_{t+1}\right)$ is $(k+1) \times(k+1)$. Consequently, if there exists a $s_{t+1} \subset S^{(t)}$, it can be clearly seen that the monotone function $F$ satisfies

$$
F\left(s_{t+1}\right)+F\left(s_{t+1}^{\prime}\right) \geq F\left(s_{t+1}^{\prime} \cup s_{t+1}\right)+F\left(s_{t+1}^{\prime} \cap s_{t+1}\right) .
$$

As illustrated by Fujishige in [31], $F\left(s_{t+1}\right)$ is submodular.

Now, utilizing the fundamental results in [30], we can state that our near-optimal solution addressed by the greedy algorithm is bounded within $1-(1 / e)$ of the optimal solution. That is,

$$
F\left(\bar{s}_{t+1}\right) \geq\left(1-\frac{1}{e}\right) F\left(s_{t+1}^{o p t}\right) .
$$

The guarantee proved in this section not only shows that the proposed algorithm has a lower bound of $1-(1 / e)$ as compared to the optimal solution but also demonstrates that an upper bound can be obtained from our near-optimal solution for any other approaches. In other words, any algorithms used to resolve the combinatorial NP-hard problem (16) is bounded by an upper bound of $\left(1-\frac{1}{e}\right) F\left(\bar{s}_{t+1}\right)$.

\section{Results AND Discussion}

In this section, we provide a numerical experiment to demonstrate the performance of the spatial prediction under consideration of data locational errors. Moreover, we illustrate the effectiveness of the proposed adaptive sampling strategy for a group of mobile wireless sensors.

In this discussion, we consider a situation where, at time step $t$, all mobile sensors make new observations and transmit them to the sink via a specific routing tree. Then the base station computes the centralized sampling strategy and sends control commands back to each robotic sensor. All predictions are estimated by the sink. Note that the experiment was implemented in two dimensional environments.

Consider a realization of the physical quantity generated within 100 units $\times 50$ units, which serves as ground truth, shown in Fig. 1. There were five $(N=5)$ mobile wireless sensors used with constrained individual displacements of a maximum radius of 5 units in every time step. All 5 robotic sensors were started from the pre-defined locations. We have assumed the knowledge of the system error $\sigma_{\text {sys }}^{2}=0.25$. The spatial field was discretized into 10000 small areas, which were considered as spatially interested sites. Therefore, the sampling paths were designed on a 10000 lattice. Notice that the lattice can be regular or irregular. The mean parameters and the hyperparameters are estimated online at each time step. We then computed the predicted field and the prediction 


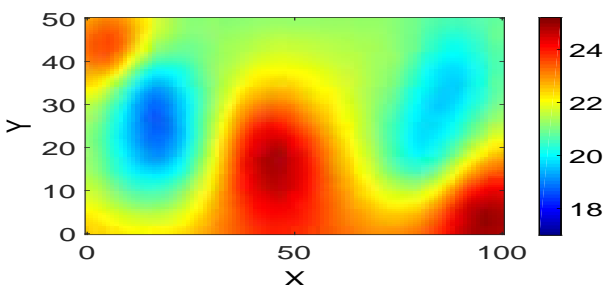

(a)

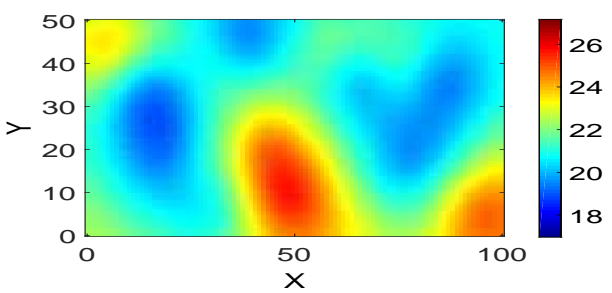

(c)

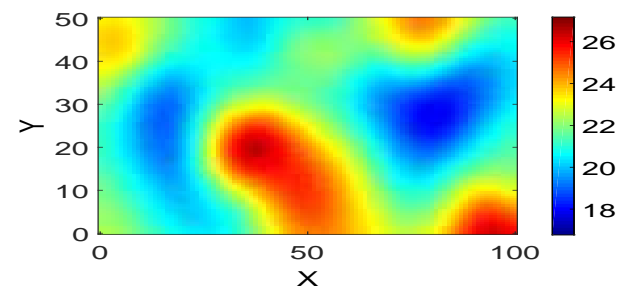

(e)

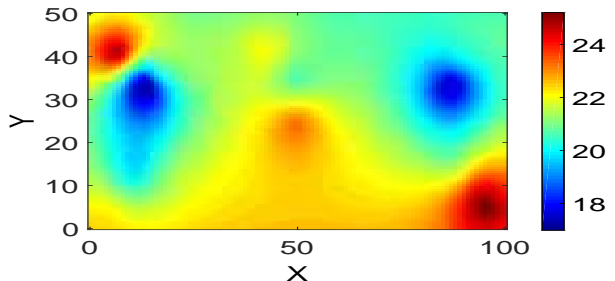

(b)

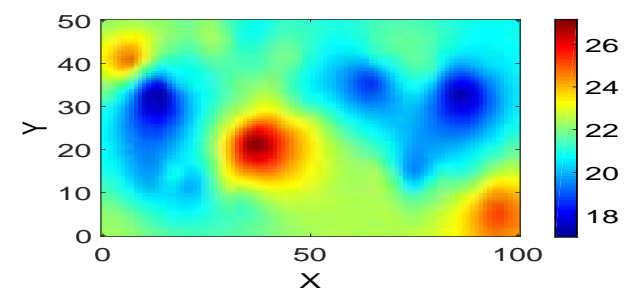

(d)

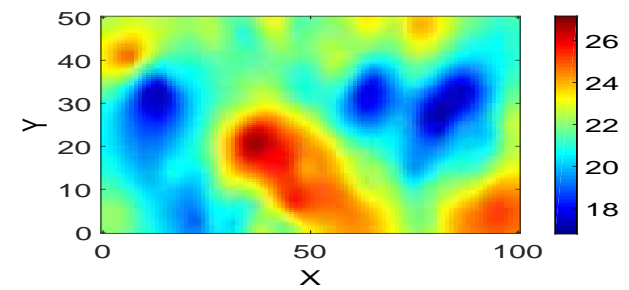

(f)

Fig. 3: Predicted fields with (left column) and without (right column) data locational errors at time steps $t=5$ (a) and (b), $t=10$ (c) and (d), and $t=20$ (e) and (f). Range of the fields is illustrated in color bars, which are kept similar in each row.

error variances in the whole environment. The computational time for achieving the efficient sampling paths for MRWSs was also analysed.

Due to imprecision of positioning measurement devices, the sensing locations contain uncertainties. We assume that these uncertainties have an uncorrelated bivariate normal distribution with a zero mean and variance $\sigma_{e}^{2}=2^{2}$. By incorporating these data locational errors into predicting the physical process at unmeasured locations, we computed the predicted means and the prediction error variances by utilizing equations (7) to (9). Figures $3 \mathrm{a}, 3 \mathrm{c}$ and $3 \mathrm{e}$ illustrate the prediction fields at time steps $t=5,10$ and 20. It can be seen that the predicted means are approaching the true field as illustrated in Fig. 1 when the number of the observations increases. White circles in Fig. 4a show the trajectories of the near-optimal paths of the mobile wireless sensors up to time $t=20$. Fig. $4 \mathrm{a}$ also shows that the closer to observed locations the unmeasured locations are, the lower their variances are.

For the purpose of comparisons, we then conducted another experiment in which the predicted fields were obtained by using the noise-less data locations and the noisy measurements. This computation was carried out based on the analysis discussed in [21]. The sampling paths for the MRWSs in this case study were near-optimally found by the strategy proposed in Section III although the errors at the sensor locations were assumed zero. The prediction results corresponding to ignoring the errors in the measurement locations are shown in the right columns of Fig. 3 for the mean values and Fig. 4 for the variance values. Comparing two columns in Fig. 3 , generally, the surfaces of the predicted fields under the presence of the data locational errors are smoother than those obtained without considering these location uncertainties. It can also be clearly seen that the prediction means in Figures $3 \mathrm{~b}, 3 \mathrm{~d}$ and $3 \mathrm{f}$ are more highly comparable with the ground truth in Fig. 1 than Figures 3a, 3c and 3e. Furthermore, the prediction variances are more sensitive to the sensing location errors than the prediction means. As demonstrated in Fig. 4, in the same scale of color bars, the surface of the predicted variances in Fig. 4a is worse than that in Fig. 4b. Further, in Fig. 4a the variances at the sensor locations are greater than zero, which is not natural if the prediction is experimented at the true locations as shown in Fig. 4b. This dissimilarity is seriously caused by the presence of the uncertainties at the measurement locations, which also means the network does not know exactly where the mobile agents are located. On the other hand, let us consider the near-optimal sampling paths for the robotic sensors obtained by the proposed greedy algorithm in Fig. 4. Even in two case studies of with and without incorporating the locational errors into the sampling path optimization problem both the networks of 5 mobile sensors start at the same pred-defined starting conditions, the resulting navigations from time instant $t=2$ to time instant 


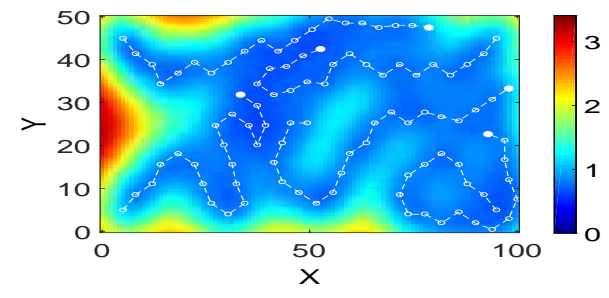

(a)

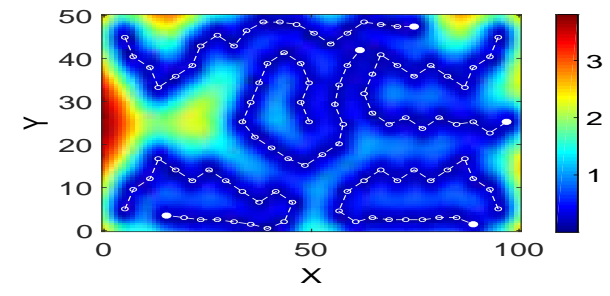

(b)

Fig. 4: The predicted variances in the fields at time $t=20$ with (a) and without (b) location errors. The sampling paths for MRWSs up to time step $t=20$ are illustrated by white circles, where current mobile sensor locations are shown in white dots. Range of the variances is illustrated in color bars in the same scale.

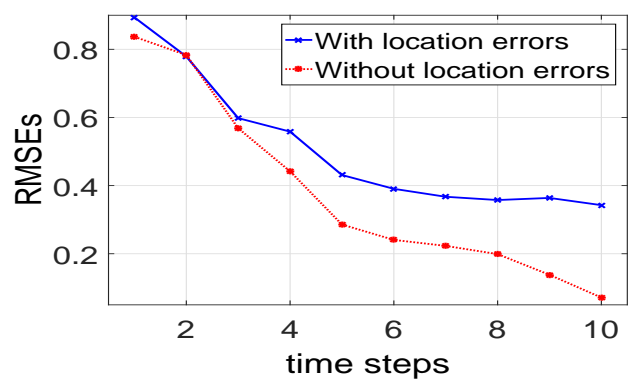

Fig. 5: The results of the Intel Lab data set. Root mean square errors

$t=20$ are completely different. The comparative distinctions of the resulting prediction variances and the sampling paths in Fig. 4 as well as the predicted means in Fig. 3 illustrate the significant effect of the presence of the errors in the sensing locations on the spatial prediction in the mobile sensor networks.

Another important aspect to compare is the computational complexity in finding the near-optimal sampling paths for the robotic sensors. As analysed in Section III, the informative sampling paths for the MRWSs are formulated to minimize the average of the predicted variances at all unobserved locations, which initially leads to the optimization problem (15). Nevertheless, this issue is then proved to be computationally expensive. An equivalent but efficient optimality criterion is proposed and formulated in (16). Theoretically, the new proposition is practically feasible. Let us investigate both these navigation optimality criteria in this particular illustrative experiment. It is to be noted that since the covariance and cross-covariance matrices are dependent on the measurements, the run time of the algorithms slightly ascend with the rise of the number of observations. Take an example, at time step $t=10$, the number of the measurements collected is 50 , our proposed algorithm took approximately 90 seconds to address the problem (16), whereas the solution of the original problem (15) required approximately 6 hour run time, implemented on R V3.0 with a PC of $3.1 \mathrm{GHz}$ Intel Core i5-2400 Processor.

In order to show consistency of the proposed method, in this section, we also implemented our algorithm in a realworld temperature data set from the Intel Berkeley Research
Lab [32]. There were ten $(N=10)$ mobile sensors visually deployed in the region of $40.5 \mathrm{~m} \times 31 \mathrm{~m}$ to navigate through the environment to collect temperature values. The locational errors in this implementation were assumed to have an uncorrelated bivariate normal distribution with a zero mean and a variance of $1^{2}$. We investigated the root mean square errors (RMSEs), which were computed based on the predicted values and the actual measurements at spatial locations of interest. As expected, the prediction errors under considering the locational errors are worse than those obtained by an assumption of the true locations. Especially, the more measurements are collected, the more serious the effect of the presence of the sensor location errors on the prediction results is. The reason is that there is more location uncertainties incorporated into the prediction. Furthermore, the running time to address the problem (16) in this scenario at time step of 10 is approximately 125 seconds, implemented on the aforementioned PC. It is apparent that computational time of the proposed method grows with an increased number of observations. However, it is noted that the computing time is practical since the network may stop collecting data as soon as the prediction accuracy is reached. For instance, in the realistic scenario implementation, the RMSEs quickly decline below 0.1 after 10 time instants.

\section{CONCLUSION}

The paper has presented an efficient method for the MRWSNs to monitor and predict the GP based spatial physical processes, where data locational errors are integrated into the formulas rather than assuming that sensor locations are true. In the proposed technique, a sampling optimization criterion that aims to minimize the average variance is presented to optimally find the paths for the mobile agents. Though this navigation problem is intrinsically NP-hard, solving it by a greedy algorithm is proved to be practically feasible for the energy-constrained MRWSNs. The obtained solutions are then proved to be guaranteed by a level of the performance as compared with the optimum. The proposed approach is finally evaluated on numerically generated and real-life data sets in which effect of localization uncertainties on prediction results is clearly illustrated. Limitation of the paper is the fact that the network may be influenced by delay in data transmission. We are intending to enhance this issue and implement the algorithm in the realistic tests in the future research works. 


\section{REFERENCES}

[1] N. E. Leonard, D. Paley, F. Lekien, R. Sepulchre, D. M. Fratantoni, and R. Davis, "Collective motion, sensor networks and ocean sampling," Proceedings of the IEEE, vol. 95(1), pp. 48-74, 2007.

[2] M. Mysorewala, D. Popa, and F. Lewis, "Multi-scale adaptive sampling with mobile agents for mapping of forest fires," Journal of Intelligent and Robotic Systems, vol. 54(4), pp. 535-565, 2009.

[3] D. V. Le, H. Oh, and S. Yoon, "Environment learning-based coverage maximization with connectivity constraints in mobile sensor networks," IEEE Sensors Journal, vol. 16(10), pp. 3958-3971, 2016.

[4] J. Binney, A. Krause, and G. S. Sukhatme, "Optimizing waypoints for monitoring spatiotemporal phenomena," The International of Robotics Research, vol. 32(8), pp. 873-888, 2013.

[5] J. Choi, J. Lee, and S. Oh, "Swarm intelligence for achieving the global maximum using spatio-temporal Gaussian processes," in Proc. American Control Conference, Washington, USA, June 2008, pp. 135-140.

[6] W. Wu and F. Zhang, "Robust cooperative exploration with a switching strategy," IEEE Transactions on Robotics, vol. 28(4), pp. 828 - 839, 2012.

[7] R. Graham and J. Cortes, "Spatial statistics and distributed estimation by robotic sensor network," in Proc. IEEE American Control Conference, Baltimore, MD, USA, 2010, pp. 2422-2427.

[8] C. E. Rasmussen and C. K. I. Williams, Gaussian processes for machine learning. The MIT Press, Cambridge, Massachusetts, London, England, 2006.

[9] J. Suh and S. Oh, "Efficient environmental monitoring using cost-aware path planning," in Proc. 13th International Conference on Control, Automation and Systems, Gwangju, Korea, 2013, pp. 1362-1365.

[10] J. Cortés, "Distributed Kriged Kalman filter for spatial estimation," IEEE Transactions on Automatic Control, vol. 54, pp. 2816-2827, 2009.

[11] Y. Xu and J. Choi, "Adaptive sampling for learning Gaussian processes using mobile sensor networks," Sensors, vol. 11, pp. 3051-3066, 2011.

[12] Y. Xu, J. Choi, S. Dass, and T. Maiti, "Sequential Bayesian prediction and adaptive sampling algorithms for mobile sensor networks," IEEE Transactions on Automatic Control, vol. 57, pp. 2078-2084, 2012.

[13] R. Marchant and F. Ramos, "Bayesian optimization for intelligent environmental monitoring," in Proc. IEEE/RSJ International Conferece on Intelligent Robots and Systems, Algarve, Portugal, October 2012, pp. 2242-2249.

[14] A. Baggio and K. Langendoen, "Monte Carlo localization for mobile wireless sensor networks," Ad Hoc Networks, vol. 6(5), pp. 718-733, 2008.

[15] C. Ou and W. He, "Path planning algorithm for mobile anchor-based localization in wireless sensor networks," IEEE Sensors Journal, vol. 13(2), pp. 466-475, 2013.

[16] J. Rezazadeh, M. Moradi, A. S. Ismail, and E. Dutkiewicz, "Superior path planning mechanism for mobile beacon-assisted localization in wireless sensor networks," IEEE Sensors Journal, vol. 14(9), pp. 30523064, 2014.

[17] X. Ma, M. Zhou, Y. Li, and J. Tan, "Intelligent mobility assisted mobile sensor network localization," in Proc. IEEE International Conferece on Robotics and Automation, Hong Kong, China, June 2014, pp. 1276 1281.

[18] G. Karthiga, C. Preethi, and R. D. H. Devi, "Localization in wireless sensor network based on mobile anchor and chord selection," in Proc. IEEE International Conference on Communication and Network Technologies, Sivakasi, India, December 2014, pp. 124-128.

[19] L. V. Nguyen, S. Kodagoda, R. Ranasinghe, and G. Dissanayake, "Mobile robotic wireless sensor networks for efficient spatial prediction," in Proc. 2014 IEEE/RSJ International Conference on Intelligent Robots and Systems, Chicago, IL, USA, September 2014, pp. 1176-1181.

[20] S. Martínez, "Distributed interpolation schemes for field estimation by mobile sensor networks," IEEE Transactions on Control Systems Technology, vol. 18, pp. 491-500, 2010.

[21] L. V. Nguyen, S. Kodagoda, R. Ranasinghe, and G. Dissanayake, "Information-driven adaptive sampling strategy for mobile robotic wireless sensor network," IEEE Transaction on Control Systems Technology, vol. 24(1), pp. 372-379, 2016.

[22] J. Gabrosek and N. Cressie, "The effect on attribute prediction of location uncertainty in spatial data," Geographical Analysis, vol. 34(3), pp. 262-285, 2002.

[23] J. P. Chilès and P. Delfiner, Geostatistics: Modelling spatial uncertainty. Wiley, 1999.

[24] N. Cressie and J. Kornak, "Spatial statistics in the presence of location error with an application to remote sensing of the environment," Statistical Science, vol. 18(4), pp. 436-456, 2002.
[25] G. S. Fishman, Monte Carlo - concepts, algorithms and applications. Springer, 1996.

[26] P. J. Diggle and P. J. Ribeiro, Model-based geostatistics. Springer, New York, USA, 2007

[27] F. Bullo, J. Cortes, and S. Martínez, Distributed control of robotic networks. Princeton University Press, 2009.

[28] C. Ko, J. Lee, and M. Queyranne, "An exact algorithm for maximum entropy sampling," Operations Research, vol. 43, pp. 684-691, 1995.

[29] R. A. Horn and C. R. Johnson, Matrix analysis. Cambridge University Press, 1985.

[30] G. L. Nemhauser, L. A. Wolsey, and M. L. Fisher, "An analysis of approximation for maximizing submodulor set functions - I," Mathematical Programming, vol. 14(1), pp. 265-294, 1978.

[31] S. Fujishige, Submodular functions and optimization. Elsevier, 2005.

[32] P. Bodik, C. Guestrin, W. Hong, S. Madden, M. Paskin, and R. Thibaux, "Intel lab data," 2004. [Online]. Available: http://db.csail.mit.edu/labdata/labdata.html

Linh V. Nguyen (M'15) received Ph.D. degree in engineering from University of Technology Sydney (UTS), Australia in 2015. He was a Research Associate at UTS in 2015 and a Research Fellow at Nanyang Technological University, Singapore in 2016. Currently, he is a Postdoctoral Research Fellow at Centre for Autonomous Systems, UTS. He is a regular reviewer of top IEEE journals and conferences. His current research interests include adaptive sampling methods for spatial prediction, signal processing, machine learning, sensors and robotics.

Sarath Kodagoda (M'10) received B.Sc.Eng.Hons. degree in 1995, specializing in Electrical Engineering, from the University of Moratuwa, Sri Lanka. He received his M.Eng. (2000) and Ph.D. (2004) degrees specializing in robotics from the Nanyang Technological University, Singapore. Before joining the ARC Centre for Autonomous Systems (CAS) at University of Technology, Sydney (UTS), he worked as a Design Engineer in a reputed multinational company. He is currently an Associate Professor and Coordinator of the Mechanical and Mechatronics program at the University of Technology, Sydney. His main research contributions are in the areas of autonomous road vehicles and Human Robot Interaction. He is an Associated Editor, Programme Committee member and a regular reviewer of a number of top robotics journals and conference proceedings. His research interests include infrastructure robotics, human robot Interaction, machine learning, perception, target tracking and mobile robotics.

Ravindra Ranasinghe (M'97) received B.Sc.Eng.Hons. degree in 1995, specializing in Computer Science and Engineering, from the University of Moratuwa, Sri Lanka. He received his Ph.D. (2002) degrees specializing in wireless communication protocols from the University of Melbourne, Australia. He is currently a Senior Research Fellow at the Centre for Autonomous Systems, University of Technology Sydney. Before joining University of Technology Sydney, he worked in several technology start-up companies in USA, Australia and Sri Lanka. His research interests include wireless communication protocols, wireless sensor networks, mobile robotics wireless sensor networks, machine learning and assistive robotics.

Gamini Dissanayake (M'06) is the James N Kirby Professor of Mechanical and Mechatronic Engineering at University of Technology Sydney (UTS). He leads the UTS Centre for Autonomous System; a team of fifty staff and students working in Robotics. He graduated in Mechanical and Production Engineering from the University of Peradeniya, Sri Lanka. He received his M.Sc. in Machine Tool Technology and Ph.D in Mechanical Engineering from the University of Birmingham, England. He taught at University of Peradeniya, Sri Lanka, National University of Singapore and University of Sydney before joining UTS in 2002. His team has an extensive track record of industry collaboration on a range of robotic systems with partners including NSW Roads and Maritime Services, Patrick Technology, Pempek Systems, Burwell Technology, Meat and Livestock Australia and Sydney Water. 